\title{
Membranas de fibra oca de diversos materiais e suas aplicações
}

\author{
Hollow fiber membranes of several materials and their applications \\ Membranas de fibra hueca de diferentes materiales y sus aplicaciones
}

Eduardo da Silva Barbosa Ferreira

ORCID: https://orcid.org/0000-0002-2670-2794

Universidade Federal de Campina Grande, Brasil E-mail: eduardosbf95@gmail.com

Rodholfo da Silva Barbosa Ferreira ORCID: https://orcid.org/0000-0002-5547-994X Universidade Federal de Campina Grande, Brasil E-mail: rodholfoferreira@gmail.com Carlos Bruno Barreto Luna

ORCID: https://orcid.org/0000-0002-2441-7439 Universidade Federal de Campina Grande, Brasil

E-mail: brunobarretodemaufcg@hotmail.com

Edcleide Maria Araújo

ORCID: https://orcid.org/0000-0003-4906-864X Universidade Federal de Campina Grande, Brasil E-mail: edcleide.araujo@ufcg.edu.br

Hélio de Lucena Lira

ORCID: https://orcid.org/0000-0002-1527-9935

Universidade Federal de Campina Grande, Brasil E-mail: helio.lira@ufcg.edu.br

\begin{abstract}
Resumo
Membrana pode ser definida como uma barreira, que separa duas fases e restringe o transporte de vários produtos químicos de forma seletiva, apresentando diferentes classificações, morfologias e geometrias. Esses materiais são utilizados em aplicações como: ultrafiltração, microfiltração, pervaporação, separação de gases, órgãos artificiais e outros fins médicos. Membranas sintéticas de fibra oca, obtidas com os diferentes tipos de materiais, vêm ganhando grande atenção nas últimas décadas devido às vantagens em relação à sua grande área específica e a maior compacidade em relação às membranas planas e tubulares utilizadas nos diversos processos de separação com membranas. As fibras ocas em sua grande maioria são preparadas por meio do método de inversão de fases, através da técnica de extrusão a frio com imersão por precipitação. O número de membranas poliméricas disponíveis comercialmente é maior quando comparado com os outros materiais, devido principalmente ao seu baixo custo, mas as membranas provenientes de materiais inorgânicos vêm ganhando uma maior atenção devido a propriedades como resistência química e térmica. Assim, o seguinte estudo tem como objetivo apresentar as principais características das membranas de fibra oca de diversos materiais, destacando as principais vantagens, os métodos de obtenção e aplicações.
\end{abstract}

Palavras-chave: Membrana; Fibra oca; Inversão de fases; Aplicações.

\begin{abstract}
Membrane can be defined as a barrier, separating two phases and restricts the transport of various chemicals selectively, showing different classifications, morphologies, and geometries. These materials are used in applications such as: ultrafiltration, microfiltration, pervaporation, gas separation, artificial organs and other medical purposes. Hollow fiber synthetic membranes obtained from different types of materials have been gaining a great attention in last decade because the advantages in relation to its large area available of the membranes and the greater compactness relative flat and tubular membranes used in the various membrane separation processes. Hollow fibers are mostly prepared by the phase inversion method by cold extrusion technique with precipitation immersion. The number of polymeric membranes available commercially is higher when compared to other materials, due mainly to its low cost, but the membranes from inorganic materials have been gaining greater attention due to properties such as thermal and chemical resistance. Thus, the following study aims to present the main characteristics of hollow fiber membranes of different materials, highlighting the main advantages, the methods of obtaining and applications.
\end{abstract}

Keywords: Membrane; Hollow fiber; Phase inversion; Applications. 


\section{Resumen}

La membrana se puede definir como una barrera, que separa dos fases y restringe selectivamente el transporte de diversos productos químicos, presentando diferentes clasificaciones, morfologías y geometrías. Estos materiales se utilizan en aplicaciones tales como: ultrafiltración, microfiltración, pervaporación, separación de gases, órganos artificiales y otros fines médicos. Las membranas de fibras sintéticas huecas, obtenidas con diferentes tipos de materiales, han ganado una gran atención en las últimas décadas debido a las ventajas en relación a su gran superficie específica y a la mayor compacidad en relación a las membranas planas y tubulares utilizadas en los distintos procesos de separación con membranas. La gran mayoría de fibras huecas se preparan mediante el método de inversión de fase, utilizando la técnica de extrusión en frío con inmersión por precipitación. El número de membranas poliméricas disponibles comercialmente es mayor en comparación con otros materiales, principalmente debido a su bajo costo, pero las membranas de materiales inorgánicos han ganado mayor atención debido a propiedades como la resistencia química y térmica. Así, el siguiente estudio tiene como objetivo presentar las principales características de las membranas de fibra hueca de diferentes materiales, destacando las principales ventajas, los métodos de obtención y aplicaciones.

Palabras clave: Membrana; Fibra hueca; Inversión de fase; Aplicaciones.

\section{Introdução}

No início da década de 70, juntamente com os processos de separação já consolidados como destilação, filtração, absorção, troca iônica, centrifugação, extração por solvente, cristalização e outros, houve o surgimento de uma nova alternativa que utilizavam membranas sintéticas como barreira seletiva, mostrando-se como uma possibilidade de realizar a mesma função e apresentar semelhantes características ou até melhores de seletividade e permeabilidade que a membrana natural (Habert, Borges \& Nóbrega, 2006).

Segundo Anadão (2010), membrana é uma barreira seletiva entre duas fases, que, sob ação de uma força motriz, permite a passagem de uma ou mais espécies selecionadas da fase de alimentação, a qual é uma mistura de caráter líquido, gasoso ou solução. Esta barreira pode ser de origem orgânica ou inorgânica, simples ou composta e apresenta-se na forma densa ou porosa (Shamu et al., 2019). A membrana tem, assim, a capacidade de transportar determinados componentes de maneira mais eficaz, retendo outros que fazem parte da mistura de alimentação (Mulder, 1996).

Devido à grande capacidade de substituir alguns processos tradicionais de separação, como sendo uma alternativa promissora desde os primórdios da sua utilização, os processos de separação por membranas (PSM) vem num crescimento cada vez maior nos últimos anos, sendo aplicados no processo produtivo de várias indústrias tais como a química, alimentícia, biotecnologia, farmacêutica, metalúrgica, automotiva, têxtil, bebidas, mineração, entre outras, além de tratamento de águas para usos domésticos (Galvão \& Gomes, 2015; Rodrigues et al., 2018).

Os PSM possuem diversas vantagens em relação aos processos de separação já conhecidos, sendo energeticamente mais vantajosos e a não utilização de agentes químicos, sendo uma tecnologia simples e de fácil operação, conquistando cada vez mais o espaço no setor industrial mundial, como uma técnica ambientalmente e economicamente viável, como também segura (Lima et al., 2011).

Com o aumento da competitividade no setor industrial, que cada vez mais passou a exigir uma produtividade mais elevada, levou à elaboração de diferentes configurações de membranas, como as membranas planas, tubulares e as de fibra oca. Os principais aspectos a serem considerados na seleção da geometria adequada são as variáveis do processo e as características da mistura a ser fracionada (Bertoldo, 2005; Habert, Borges \& Nóbrega, 2006). Apesar da predominância no mercado das membranas planas, sendo a que apresenta a geometria mais simples das citadas, as membranas na forma de fibra oca também têm papel fundamental no mercado, sendo utilizadas em separação de gases, ultrafiltração, pervaporação e diálise (Li et al., 2008). O que torna a membrana de fibra oca favorável para essas aplicações industriais é a sua elevada área superficial para o preenchimento adequado do volume dos módulos e suportes (Huang \& Feng, 2018).

Diante desse contexto, o principal objetivo deste trabalho é apresentar as características gerais das membranas de fibra oca de diversos materiais, destacando suas vantagens, os seus métodos de obtenção e suas aplicações. 


\section{Metodologia}

Para o estudo, foi utilizado a metodologia de pesquisa bibliográfica, de natureza qualitativa (Pereira et al., 2018), realizando buscas no banco de dados Google Acadêmico e Web of Science, utilizando os termos a seguir: Membrana/Membrane, Fibra Oca/Hollow Fiber, Membranas Poliméricas/Polymeric Membranes, Membranas Inorgânicas/Inorganic Membranes. Foram utilizadas as bibliografias onde apresentaram maior interesse para o estudo, sem exclusão de trabalhos por ano de publicação.

A presente revisão aborda um tema geral, reunindo os vários trabalhos e livros desenvolvidos sobre a produção e aplicação de membranas de fibra oca de materiais orgânicos e inorgânicos. Este estudo se trata de uma revisão temática, centrada no conceito, histórico, métodos de obtenção, e aplicações das membranas de fibra oca, sendo um trabalho de nível informativo, apresentando os diversos tipos de materiais que podem ser empregados na produção de uma membrana de fibra oca e suas principais características. Assim, a revisão apresentada é uma síntese das características, produção e aplicação de membranas de fibra oca utilizadas nos diversos ramos industriais.

\section{Resultados e Discussão}

\subsection{Histórico}

Quando se trata da história e do desenvolvimento da tecnologia de membranas, duas vertentes podem ser seguidas, que seria a científica e a comercial. Em meados do século XVIII, fenômenos com membranas foram observados e estudados primariamente para elucidar as propriedades de barreira e transporte. O aperfeiçoamento dos processos de separação por membranas e suas aplicações são considerados recentes no meio industrial (Mulder, 1996).

Em 1748 foi registrado o primeiro fenômeno relacionado a membranas pelo francês Abbé Nollet, realizando um experimento composto de um recipiente com vinho, o qual foi selado com uma bexiga de origem animal e imerso em água pura. Ele observou que a água difundia em direção ao vinho, com a bexiga estufando e chegando até mesmo a se romper, concluindo que o transporte de água ocorreria de uma solução mais diluída para uma mais concentrada, quando estas eram separadas por uma membrana semipermeável. Esta experiência evidenciou, pela primeira vez, as características de permeabilidade e seletividade de uma membrana (Habert, Borges \& Nóbrega, 2006; Anadão, 2010).

Apesar de alguns estudos na área de membranas terem ocorrido desde a década de 1930, somente na década de 1950 as pesquisas com membranas passaram a gerar maior interesse científico e comercial, quando, nos Estados Unidos, passou-se a investir em pesquisas para a dessalinização de água do mar, ocorrendo avanços com relação as técnicas de produção das membranas e aumento do fluxo permeado de água (Habert, Borges \& Nóbrega, 2006).

Entre 1958 e 1960, um avanço no que diz respeito às aplicações de membranas industriais foi atingido pelo desenvolvimento de membranas assimétricas. Loeb e Sourirajan realizaram estudos sobre a modificação das membranas já existentes, aperfeiçoando uma técnica para preparo da membrana, conhecida hoje como técnica de inversão de fase por imersão-precipitação. Estas membranas consistem em uma camada muito fina e densa suportada por uma subcamada porosa, mas muito mais permeável, que proporciona a resistência mecânica. O fluxo desta foi 10 vezes maior (sendo considerada eficiente) que o de qualquer membrana disponível e tornou um método potencialmente prático de dessalinização da água (Mulder, 1996; Baker, 2004; Habert, Borges \& Nóbrega, 2006; Anadão, 2010).

Novos métodos para disposição das membranas foram surgindo, como os módulos de fibras ocas. Em 1966, membranas de fibra oca foram desenvolvidas por Mahon, e o grupo Dow Chemical, sendo os primeiro a comercializarem a esse tipo de membrana, representando um dos principais eventos na tecnologia de membranas (Baker, 2004). Pesquisas e desenvolvimento usando uma membrana de acetato de celulose do tipo fibra oca foi conduzido pela Monsanto, Toyobo e 
outros, além da Dow Chemical. Em 1979, a Toyobo anunciou uma membrana de fibra oca de triacetato de celulose para dessalinização de água do mar (Li et al., 2008).

Sabe-se que, devido a maior facilidade de obtenção e produção, as membranas sintéticas mais utilizadas na indústria são oriundas de materiais poliméricos, apresentando características químicas e físicas variadas de acordo com o processo de obtenção e o tipo de polímero utilizado. Já os materiais inorgânicos, apresentando características como estabilidade química e térmica, maior vida útil, boa integridade estrutural e facilidade de limpeza, sendo superiores aos materiais poliméricos, recentemente vêm ganhando mais importância no mercado, tendo como grande desvantagem o custo de produção (Mulder, 1996; Habert, Borges \& Nóbrega, 2006).

Atualmente, uma grande quantidade de pesquisadores vêm dando grande importância na síntese, produção e caracterização de membranas, tanto poliméricas como inorgânicas, visando cada vez aumentar o número de opções de materiais utilizados, baratear custos utilizando resíduos, como também otimizar o processo de obtenção.

\subsection{Processo de separação por membranas (PSM)}

O processo de separação de membranas é considerado uma tecnologia emergente, sofrendo um grande e rápido crescimento nas últimas décadas. Nos últimos 55 anos, as membranas evoluíram de uma ferramenta de laboratório para uma escala industrial com impacto técnico e comercial significativo. Os processos de membranas fornecem uma técnica de separação altamente flexível para a concentração/separação seletiva de soluto e permitem a reciclagem e a reutilização (Nath, 2017).

Os PSM têm sido considerados como uma opção eficaz, devido as características especiais das membranas, que os têm tornado atraentes para aplicações industriais, como a compacidade, facilidade de fabricação, seletividade, operação e desenho modular, sendo também energeticamente mais vantajosos (Wang et al., 2015; Nath, 2017).

As separações nos processos com membrana são os resultados das diferenças nas taxas de transporte de espécies químicas através da interface da membrana. A taxa de transporte é determinada pela força motriz ou forças que atuam sobre os componentes individuais e sua mobilidade e concentração dentro da interface. A mobilidade é determinada principalmente pelo tamanho molecular do soluto e pela estrutura física do material da interface, enquanto a concentração do soluto na interface é determinada pela compatibilidade química do soluto e interface, do tamanho do soluto e da estrutura da membrana. A mobilidade e a concentração do soluto dentro da interface determinam o tamanho de um fluxo produzido por uma determinada força motriz (Porter, 1989).

O processo de separação por membranas é utilizado à décadas em várias áreas da indústria mundial, devido a sua grande capacidade, como já citado anteriormente, sendo capaz de substituir os processos clássicos, utilizados na indústria química, famacêutica, alimentícia, médica, têxtil e tratamentos de água.

\subsection{Membranas}

As membranas podem ser consideradas como uma barreira semipermeável para uma filtração em escala molecular, que separa duas fases e que restringe, totalmente ou parcialmente, a condução de uma ou várias espécies químicas (solutos) presentes na solução (Hamza et al., 1997).

O transporte da solução pode ocorrer por difusão ou convecção, sendo induzido por um gradiente de potencial químico (pressão, concentração e temperatura) ou potencial elétrico. A parcela da corrente de alimentação que permeia a membrana é conhecida como permeado, já a fração que não atravessa é chamada de concentrado ou não-permeado (Mulder, 1996; Kojuch, 2010). 
Segundo Mulder (1996), a diferença entre o processo de separação com membranas e as outras técnicas convencionais é a utilização de outra fase que é a membrana, introduzida entre o volume das duas fases envolvidas na separação, podendo originar vantagens de eficiência e seletividade. Devido ao papel significativo da membrana nos custos de operação, esforços são feitos para obter membranas econômicas (Rastegarpanah \& Mortaheb, 2016). A Figura 1 representa esquematicamente o funcionamento de uma membrana.

Figura 1. Representação esquemática de uma membrana.

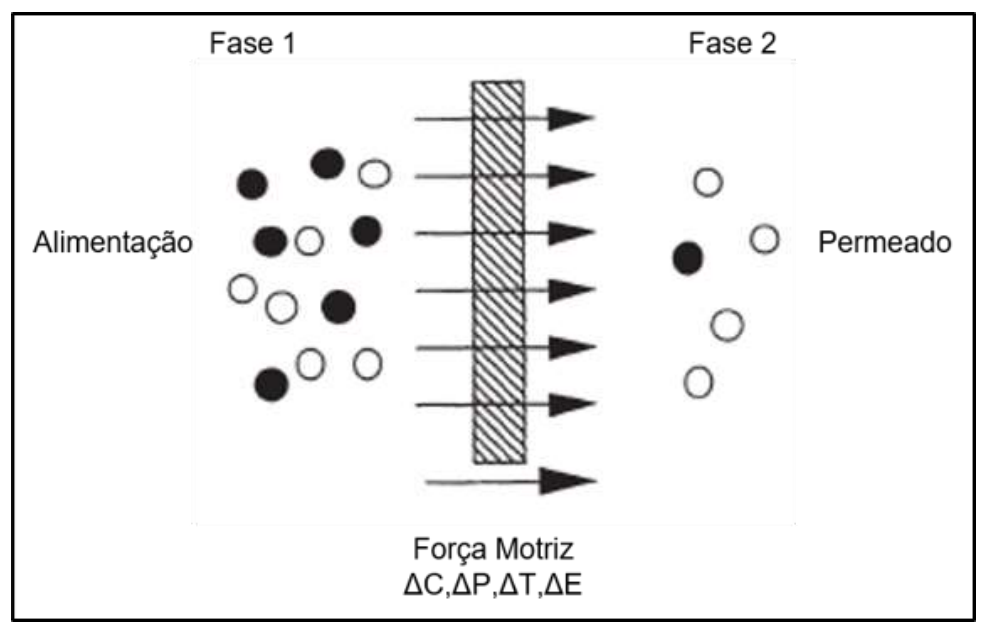

Fonte: Adaptado de Mulder (1996).

Tem-se na Figura 1 justamente o conceito de membrana, que seria uma barreira que irá separa duas fases, a alimentação (fase 1) e o permeado (fase 2), onde o permeado seria a parte da solução que não foi retida pela membrana, e o que será reaproveitado.

Na escala industrial, as membranas sintéticas são produzidas a partir de duas classes de materiais distintas: orgânicas e inorgânicas. Os materiais orgânicos, na sua maioria são formados por materiais poliméricos como poliamida, polisulfona, poliacrilonitrila, policarbonato, polieterimida, e os inorgânicos, como cerâmicas e metais (Habert, Borges \& Nóbrega, 2006).

Sabe-se que as membranas mais fabricadas e comercializadas são as membranas poliméricas. No entanto, nos últimos anos, as membranas inorgânicas apresentaram grande potencial por solucionarem dificuldades de aplicação relacionadas a outros tipos de membrana, especialmente devido à integridade estrutural quando submetidas a condições drásticas como temperatura, pressão, natureza ou pH do meio. As membranas cerâmicas, uma classe especial de membranas microporosas, estão sendo usadas em aplicações de microfiltração e ultrafiltração para as quais é necessária resistência química e estabilidade térmica. As membranas metálicas densas, estão sendo consideradas para a separação do hidrogênio das misturas de gases, e as membranas líquidas estão sendo desenvolvidas para processos de transporte (Baker, 2004; Armoa \& Jafelicci, 2011). Dependendo da sua aplicação, as membranas sintéticas apresentam diferentes estruturas. Pode-se observar na Figura 2 as estruturas morfologicas das membranas. 
Figura 2. Desenho esquemático da seção transversal de uma membrana isotrópica e anisotrópica.

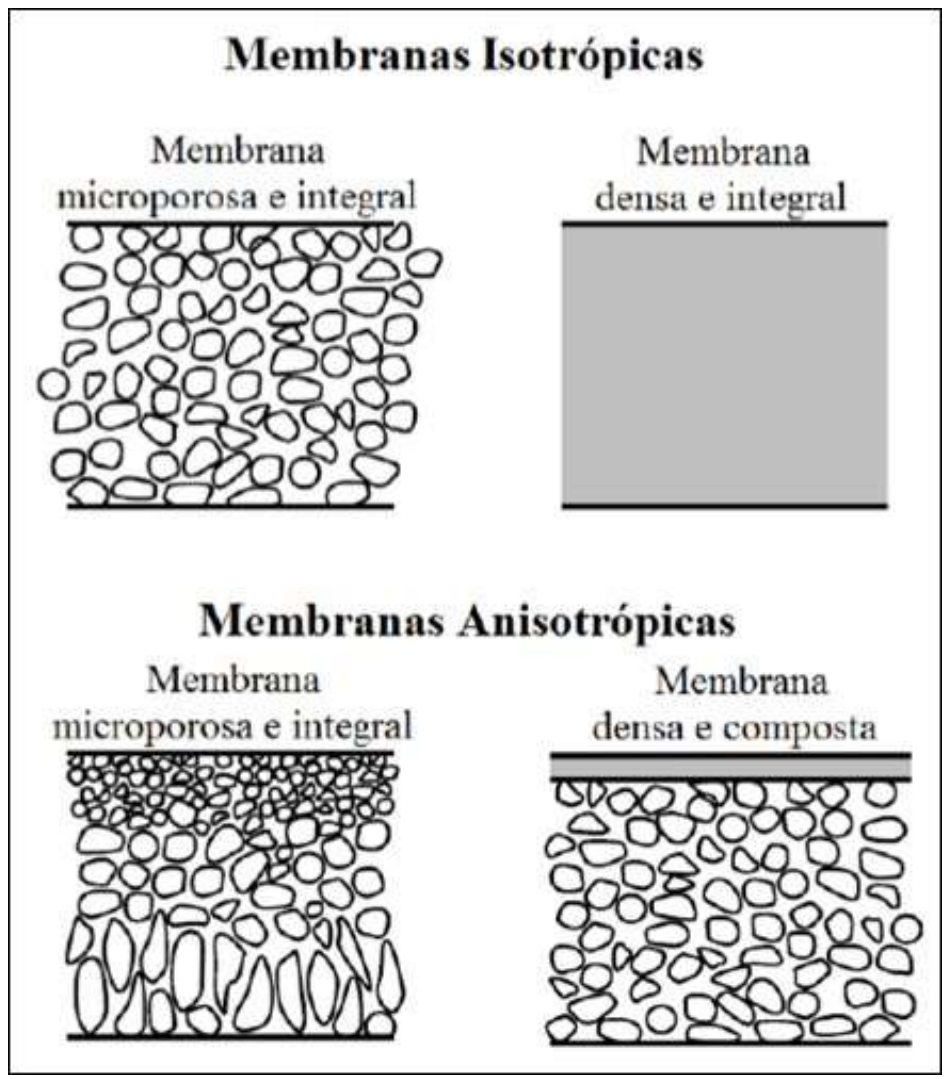

Fonte: Adaptado de Baker (2004).

As membranas isotrópicas têm uma estrutura uniforme em toda a espessura da membrana, podendo ser porosas ou ter uma estrutura uniforme bastante densa. As membranas anisotrópicas não apresentam as mesmas características morfológicas ao longo de sua espessura, e se caracterizam por uma região superior fina, mais fechada (com poros ou não), chamada de "pele", suportada em uma estrutura porosa, chamada de substrato, categorizadas em três estruturas básicas: membrana anisotrópica integral com camada porosa; membrana anisotrópica integral com camada densa e membranas compostas, quando materiais diferentes são empregados no preparo de cada região (Khulbe, Feng \& Matsuura, 2008).

\subsection{Fibra oca}

As fibras ocas têm sido utilizadas nas últimas décadas em muitas aplicações, como osmose reversa, ultrafiltração, separação de gases, órgãos artificiais, alguns outros fins médicos, e vêm recebendo grande atenção nos últimos anos devido às vantagens oferecidas por essa geometria. $\mathrm{O}$ uso de fibras ocas tornou-se popular em muitos setores industriais desde que Mahon (1966) patenteou as primeiras membranas de fibras ocas. A morfologia e o desempenho de membranas de fibra oca são funções complexas de muitos parâmetros envolvidos na sua produção (Habert, Borges \& Nóbrega, 2006; Khulbe, Feng \& Matsuura, 2008). A Figura 3 ilustra membranas do tipo fibra oca dentro de um módulo. 
Figura 3. Membranas do tipo fibra oca.

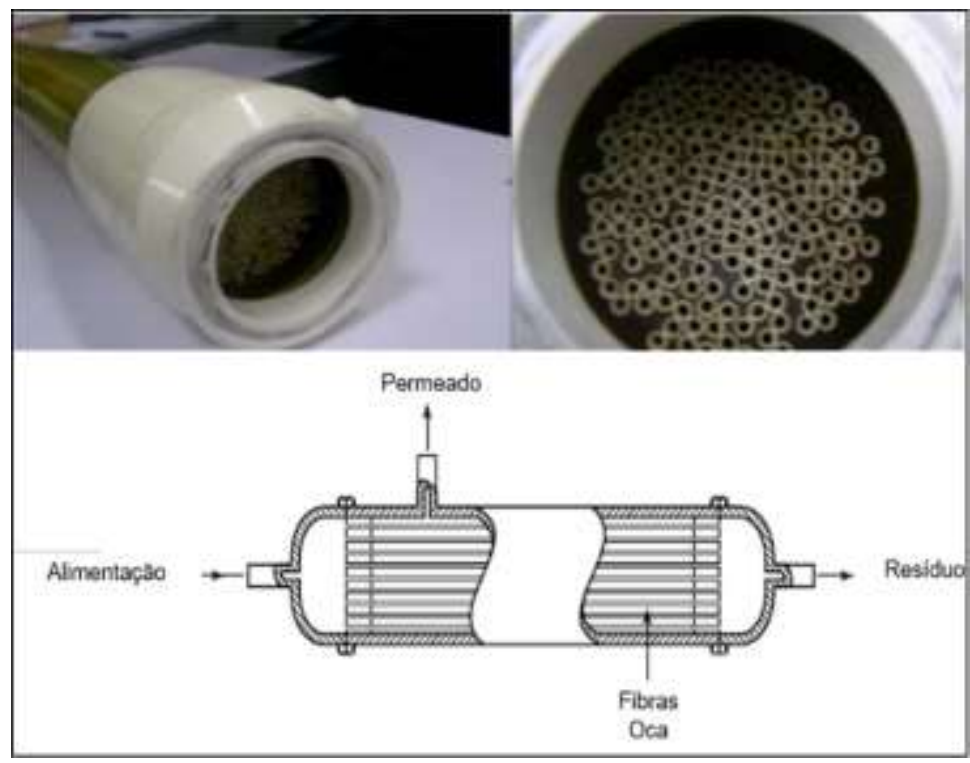

Fonte: Adaptado de Baker, (2004) e Radjenović et al., (2007).

Para as devidas aplicações, as membranas de fibra oca são utilizadas em conjunto por meio dos módulos de permeação, conforme observado na Figura 3. A quantidade de membranas no interior dos módulos varia de acordo com sua aplicabilidade. Dessa forma, o efluente é inserio no canal de alimentação, permitindo que as membranas possam tratá-lo, separando o permeado dos resíduos.

Os estudos da morfologia de fibra oca baseavam-se em imagens transversais tomadas por microscopia eletrônica de varredura (MEV), através das quais as estruturas assimétricas das membranas de fibras foram claramente vistas. Em contraste com o MEV, os estudos morfológicos de fibras ocas por microscopia eletrônica de força atômica (AFM) são principalmente baseados na imagem da superfície da fibra, tanto no interior como no exterior (Khulbe, Feng \& Matsuura, 2008).

O diâmetro das fibras ocas pode variar consideravelmente, de 50 até $3000 \mu \mathrm{m}$, dependendo da aplicação. As fibras devem ser embaladas em feixes e encapsuladas em tubos para formar um módulo de membrana. Como um módulo não deve conter fibras defeituosas ou quebradas, a produção de fibra oca requer alta reprodutibilidade e rigoroso controle de qualidade (Baker, 2004).

Os módulos de membranas de fibra oca são constituídos por um feixe de finas membranas cilíndricas dispostas no interior de um casco tubular, fixadas por uma placa na extremidade do casco. A operação dos módulos ocorre de duas maneiras, sendo elas: (i) a alimentação é introduzida no interior das fibras e o permeado é recolhido no lado do casco; (ii) a alimentação é introduzida no lado do casco e o permeado é recolhido pela extremidade da fibra. Nas duas formas de operação apresentadas, o projeto do módulo de permeação deve permitir que o escoamento da solução de alimentação e do permeado seja o mais livre possível (Maldonado, 1991; Habert, Borges \& Nóbrega, 2006).

Segundo Lavezo (2011) elencou os requisitos básicos para os módulos de membranas de fibra oca: controle do escoamento; facilidade de limpeza; elevada relação de área de membrana e volume do módulo e baixo custo de fabricação. Os tipos de membranas de fibras ocas em produção são ilustrados na Figura 4. 
Figura 4. Esquema dos principais tipos de membranas de fibras ocas.

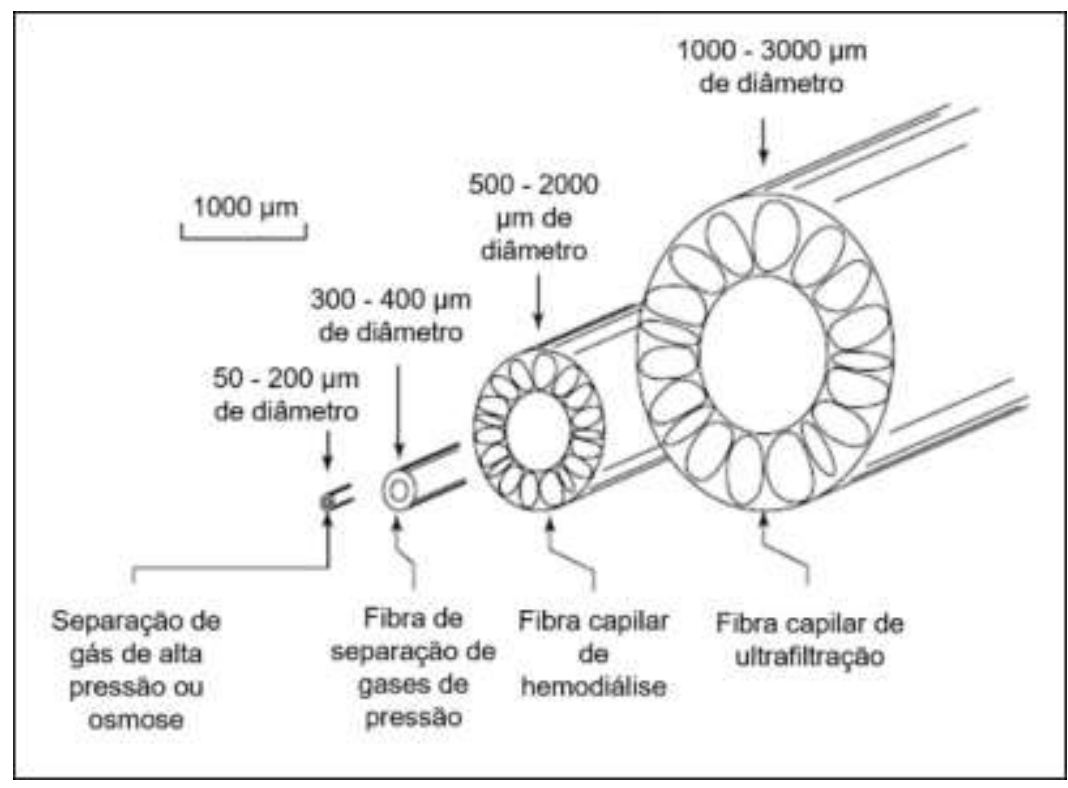

Fonte: Adaptado de Baker (2004).

Observando na Figura 4, Fibras de 50 a $200 \mu \mathrm{m}$ de diâmetro podem suportar pressões hidrostáticas muito altas aplicadas a partir do exterior, de modo que são usadas na osmose reversa ou em aplicações de separação de gases de alta pressão. Quando o diâmetro da fibra está entre 200-500 $\mu \mathrm{m}$, normalmente é utilizada para separações de gases de baixa pressão e para aplicações como hemodiálise ou ultrafiltração. Fibras com um diâmetro superior a $500 \mu$ m são chamadas de fibras capilares, utilizadas em hemodiálise e para ultrafiltração (Baker, 2004).

Uma das grandes vantagens oferecidas pela utilização da geometria do tipo fibra oca é o fato de que a relação entre a área de permeação (área da superfície da membrana) e o volume do módulo é ser bem superior à das demais geometrias, com isso tem-se uma melhor utilização do espaço e uma redução no custo de equipamento. Outra vantagem importante que as fibras ocas oferecem é o fato de serem auto suportadas, reduzindo o custo de produção do módulo de permeação. Como desvantagem, quando há a alimentação por dentro das fibras, há grande possibilidade de entupimento, e uma maior espessura da parede das fibras, que evitam o colapso em grades diferenças de pressões (Habert, Borges \& Nóbrega, 2006).

\subsection{Métodos de obtenção}

Existe um grande número de técnicas utilizadas na fabricação de membranas sintéticas. Entre os métodos disponíveis, os mais conhecidos são: sinterização, estiramento (stretching), track-etching e inversão de fases (Mulder, 1996).

As membranas de fibras ocas são geralmente obtidas através do método de inversão de fase, exibindo características físico-químicas específicas e propriedades mecânicas e de superfície geralmente favoráveis, apoiando seu uso em diversas aplicações (Madsen, 2010).

Dentre o método de inversão de fases, uma das técinicas que possibilitam uma morfologia mais variada das membranas é a extrusão a frio com precipitação por. Ela consiste em uma solução polimérica utilizando solvente escolhido de acordo com o polímero utilizado. Posteriormente essa solução é extrusada em um banho contendo um não-solvente (geralmente água), ocorrendo assim a precipitação. A Figura 5 apresenta, esquematicamente, a matriz de uma extrusora para a produção de membrana do tipo fibra oca. 
Figura 5. Esquema da matriz de uma extrusora para a produção de membranas na forma de fibra oca.

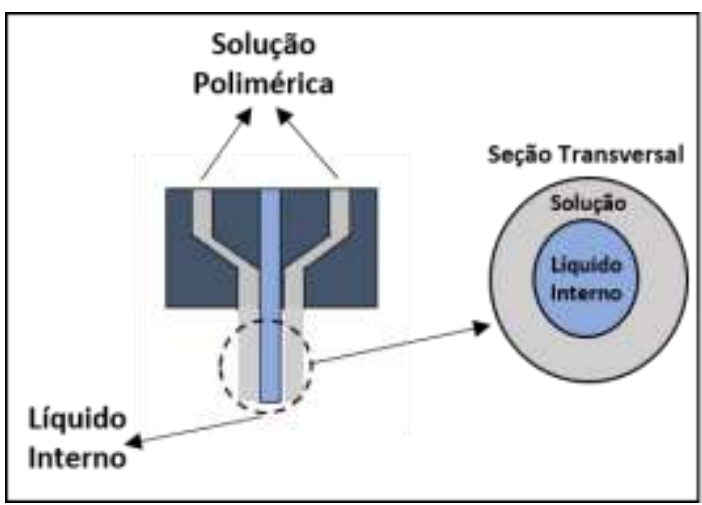

Fonte: Adaptado de Habert, Borges \& Nóbrega (2006).

A extrusora possui dois orifícios circulares concêntricos por onde escoam a solução polimérica e um líquido que visa evitar o colapso da solução, denominado de líquido interno (Habert, Borges \& Nóbrega, 2006).

O esquema de um sistema típico para a obtenção de membranas do tipo fibra oca pode ser observado na Figura 6.

Figura 6. Esquematização do sistema para obtenção de membranas do tipo fibra oca.

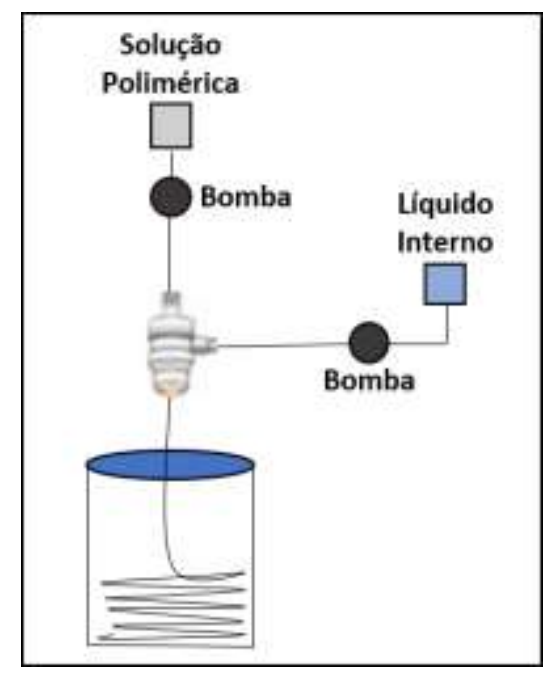

Fonte: Adaptado de Habert, Borges \& Nóbrega (2006).

Observa-se na Figura 6 que o banho de precipitação fica localizado logo a baixo da saída da extrusora, onde uma bomba promove o escoamento do líquido interno mencionado anteriormente, e outra o esvoamento da solução polimérica em direção ao banho. O processo de separação de fases, onde há a formação da estrutura da membrana, pode iniciar durante o escoamento da solução entre a matriz da extrusora e o banho de precipitação, havendo a evaporação parcial do solvente, ou a absorção de vapores de não-solvente. Após a imersão no banho, tem-se a saída do solvente e entrada do não-solvente na solução, ocasionando a precipitação e a formação da fibra oca. Por fim, a membrana é levada para o banho de lavagem e extração do solvente residual, sendo posteriormente estocada em meio aquoso (Habert, Borges \& Nóbrega, 2006).

Semelhantemente ao método de fabricação de membranas de fibra oca polimérica, este método também permite a fabricação de membranas inorgânicas. Após a solução polimérica ser preparada, a carga inorgaânica é dispersa na solução, e posteriormente, é extrusada através de uma matriz fibrilar. A fibra formada entra em contato com um banho de coagulação e é 
colocada para secar. O polímero orgânico é removido em uma atmosfera oxidante e o restante é sinterizado no forno. Com o aquecimento, os compostos voláteis saem da fibra e a superfície orgânica se torna porosa (Hilal, Ismail \& Wright, 2015).

As membranas cerâmicas de fibra oca podem ser preparadas usando um processo de três etapas com base em uma combinação de inversão de fase e métodos de sinterização envolvendo a (1) preparação de uma suspensão de fiação, (2) fiação de precursores cerâmicos de fibra oca e (3) sinterização final (Hilal, Ismail \& Wright, 2015).

Durante a produção das fibras há vários parâmetros que são conhecidos por afetar a morfologia, as propriedades e o desempenho das fibras, nos quais podem ser divididos em quatro categorias (Hilal, Ismail \& Wright, 2015):

- Parâmetros conectados à composição da solução incluem o tipo de polímero e aditivos, concentração, viscosidade e temperatura;

- Os parâmetros relacionados à fiação, incluindo temperatura, taxa de extrusão, tipo de fiação (dupla ou tripla), geometria e dimensões, comprimento do intervalo de ar e atmosfera (umidade);

- Os parâmetros relacionados à coagulação incluem temperatura, composição e taxa de injeção do fluido, e temperatura e composição do banho de coagulação;

- Os parâmetros conectados aos eventuais pós-tratamentos incluem a velocidade de alongamento, tratamentos químicos e térmicos, e técnicas de secagem.

\subsection{Fibra oca polimérica}

\subsubsection{Características gerais}

Desde Mahon (1966), houveram inúmeros desenvolvimentos no uso de acetato de celulose (CA), triacetato de celulose (CTA) e outros derivados de celulose para tornar as membranas adequadas para a dessalinização de água do mar, água salobre e outras aplicações. Desde então, outros polímeros sintéticos também foram utilizados para diversos usos. A Tabela 1 mostra os principais polímeros utilizados para a preparação de membranas de fibra oca.

Tabela 1. Polímeros mais utilizados na fabricação de membranas de fibra oca.

\begin{tabular}{cc}
\hline Polímero & Sigla \\
\hline Polietersulfona & PES \\
Polisulfona & PSU \\
Polipropileno & PP \\
Poliacrilonitrila & PAN \\
Acetato de Celulose & CA \\
Fluoreto de Polivinilideno & PVDF \\
\hline
\end{tabular}

Fonte: Autores.

As membranas poliméricas apresentam como vantagem a relativa facilidade de formação e fabricação, o que permite a obtenção de altas taxas de permeabilidade. Além disso, as membranas poliméricas têm um custo menor, quando comparados com as membranas que utilizam materiais inorgânicos. No entanto as membranas poliméricas possuem várias limitações, como 
uma baixa seletividade, instabilidade a altas temperaturas, dilatação e decomposição em solventes, que limitam o uso dessas membranas em diversas aplicações industriais (Anadão, 2010).

\subsubsection{Aplicações}

Várias membranas de fibras ocas poliméricas são preparadas e utilizadas amplamente devido as suas características. Como a primeira membrana de fibra oca polimérica foi patenteada como um dispositivo de separação, a pesquisa sobre membranas de fibra oca polimérica recebeu atenção mundial tanto da academia como da indústria, e as membranas de fibra oca feitas de diferentes materiais poliméricos têm sido progressivamente utilizadas em vários processos e aplicações de separação (Tan, Liu \& Li, 2001; Hilal, Ismail \& Wright, 2015).

Os módulos de fibras ocas são aplicados em maior escala em sistemas de MF e UF, apresentando área de membrana por volume de módulo de cerca de $1000 \mathrm{~m}^{2} / \mathrm{m}^{3}$. Essa elevada relação entre a área de permeação e o volume do módulo constitui uma vantagem para as membranas de fibras ocas, uma vez que há melhor utilização do espaço resultando numa redução do custo do equipamento (Schneider \& Tsutiya, 2001).

Além de microfiltração e ultrafiltração, as membranas de fibras ocas poliméricas vêm sendo usadas em diversas aplicações, tais como, separação de gases, órgãos artificiais e outros fins médicos (Khulbe, Feng \& Matsuura, 2008).

Segundo Mack et al. (2009) citado por Khulbe \& Matsuura (2016), outras aplicações deste tipo de membrana estão em vários estágios de desenvolvimento, por exemplo, bio-separação e biorreatores na indústria bioquímica, separação de hidrocarbonetos por pervaporação (PV) no setor petroquímico e petrolífero, e concentração e purificação de componentes valiosos na indústria de alimentos. Além disso, as membranas de fibra oca poliméricas têm sido amplamente utilizadas como armação para proliferação celular e diferenciação de tecidos que requerem uma forma tubular, por exemplo, intestino, uretra e vasos sanguíneos ou nervos.

\subsection{Fibra oca inorgânica}

\subsubsection{Caracteristicas gerais}

A combinação de alta resistência química e térmica tornou as membranas inorgânicas uma alternativa atrativa para as membranas poliméricas em geral. Devido às altas proporções superficiais/volumétricas alcançadas pela geometria da fibra oca, a compacidade dos sistemas de membranas inorgânicas de fibra oca pode exceder em grande parte a dos outros sistemas de membrana (Drioli \& Giorno, 2010). No entanto, a aplicação generalizada e em larga escala de fibras inorgânicas é dificultada, em particular, pelas suas propriedades mecânicas. O conhecimento detalhado dessas propriedades é necessário para uma comparação aceitável entre fibras diferentes, e a quantificação do comportamento de falha das fibras é de importância fundamental para o projeto e construção de sistemas multi-fibra de grande área (De Wit, Van Daalen \& Benes, 2017).

As membranas inorgânicas apresentam uma vida útil maior do que a das membranas poliméricas, podendo durar de 10 a 14 anos, uma vez que toleram regimes agressivos de limpeza. As formas de limpeza das membranas podem ser físicas e químicas. As físicas são dependentes de forças mecânicas para remoção das partículas acumuladas na superfície da membrana. Incluem a lavagem na direção do fluxo (flushing) e/ou flushing reverso e retrolavagem. Os métodos químicos dependem de reações químicas, que quebram as forças que ligam as substâncias aderidas na superfície da membrana. As formas de limpeza química são a hidrólise, digestão, saponificação, solubilização e dispersão (Lautenschlager et al., 2009).

Além do alto custo inerente à produção das membranas inorgânicas, a sua pobre resistência mecânica, caracterizada pela alta fragilidade do material, restringe a sua aplicabilidade em grande escala, mas se esse problema for solucionado, acredita-se que, no futuro, o primeiro lugar no mercado de processo de separação de membranas seja das membranas inorgânicas (Anadão, 2010). 
Níquel, ligas de níquel, óxido de níquel, misturas de óxido de ferro, alumina, alumina $\beta$, vidro, mulita, carbeto de silício e sílica são os mais materiais que têm sido mais utilizados na preparação e fabricação de fibras ocas inorgânicas, como também na área de pesquisa (Anadão, 2010).

As membranas cerâmicas de fibra oca, fabricadas através do processo de inversão de fase, consistem normalmente em duas estruturas básicas: fingers e sponges. Kingsbury e Li (2009) estudaram experimentalmente as morfologias de membranas cerâmicas de fibras ocas, preparadas a partir de suspensões de $\mathrm{Al}_{2} \mathrm{O}_{3}$, NMP (N-metil-2-pirrolidona) e polietersulfona (PES) utilizando um processo de fiação/sinterização a seco. Os resultados indicaram que podem ser esperados dois tipos de morfologias da membrana, isto é, estruturas semelhantes a fingers e sponges. Acredita-se que a formação de vazios em forma de fingers em membranas cerâmicas assimétricas é iniciada por precipitação viscosa hidrodinamicamente instável desenvolvida quando um fluido menos viscoso (não solvente) está em contato com um fluido de viscosidade mais alta (suspensão cerâmica contendo aglutinante de polímero reversível). O crescimento vazio semelhante a fingers ocorre apenas abaixo de uma viscosidade crítica da suspensão, acima da qual é observada uma estrutura semelhante a sponges em toda a seção transversal da fibra oca.

\subsubsection{Aplicações}

As membranas inorgânicas tornaram-se um tipo importante de membranas devido às suas propriedades específicas em comparação com as membranas poliméricas. O limite de temperatura superior das membranas poliméricas nunca excederá $500^{\circ} \mathrm{C}$, mas materiais inorgânicos tais como cerâmicas (carbeto de silício, óxido de zircônio, alumina, óxido de titânio) podem suportar temperaturas muito altas e são muito adequados para serem aplicados em ambientes agressivos, como por exemplo em aplicações de alta temperatura em ambientes com larga faixa de $\mathrm{pH}$, realizando uma variedade de separações de gases, como a remoção de gás ácido de fluxos de gás sintético encontrados em sistemas de geração de energia a carvão e recuperação de hidrogênio na produção petroquímica (Way \& Roberts, 1992; Mulder, 1996).

A capacidade de operar a altas temperaturas e pressões, e em ambientes corrosivos, permite que as membranas cerâmicas sejam usadas em uma gama de aplicações, incluindo filtração para fluidos corrosivos, reatores de membrana de alta temperatura, células de combustível de óxido sólido e contactores de membrana. Em particular, a natureza resistente aos solventes dos materiais das membranas cerâmicas os torna extremamente úteis para fins de filtração de solventes, pois podem ser usados com uma variedade de solventes tanto polares como apolares (Kingsbury \& Li, 2009).

As membranas cerâmicas que possuem óxidos mistos e a condução eletrônica têm atraído muita atenção devido ao potencial de sua utilização na separação do ar e na oxidação seletiva de hidrocarbonetos leves para o gás de síntese e outros produtos. As membranas cerâmicas de fibra oca se tornaram uma alternativa promissora para essa aplicação devido à sua maior área de membrana por unidade de volume (Liu \& Gavalas, 2005).

Além das aplicações citadas, os processos de filtração com membranas inorgânicas de fibra oca no tratamento de águas tornaram-se uma tecnologia mais atrativa nos últimos anos como uma possível alternativa para os processos convencionais, principalmente, no tratamento de vários tipos de águas residuais: águas residuais de algumas indústrias, como eletrônicos, petroquímicos, tratamento de resíduos radioativos, efluentes contendo matéria orgânica natural, metais pesados e águas contendo pesticidas e herbicidas (Silva, 2015).

\section{Estado da Arte}

Diante do que foi visto, alguns trabalhos sobre membranas do tipo fibra oca, de diferentes tipos de materiais poliméricos e inorgânicos foram investigados. É observado no Quadro 1 que a grande maioria das membranas são utilizando cargas inorgânicas, a fim de aumentar a porosidade e resistência mecânica das fibras, como também o apelo ambiental, onde 
muitas vezes são utilizados resíduos, como no caso de argilas, a fim de baratear custos. Assim, tem-se na Tabela 2 alguns trabalhos de membranas poliméricas e membranas com a adição de cargas inorgânicas reportados na literatura, evidenciando o tipo de membrana, o principal objetivo e principais resultados alcançados.

Quadro 1. Trabalhos de membranas de fibra oca reportados na literatura, evidenciando o tipo de membrana, o principal objetivo e principais resultados alcançados.

\begin{tabular}{|c|c|c|c|}
\hline Autores & Tipo de Membrana & Objetivo & Principais Resultados \\
\hline $\begin{array}{c}\text { Tan et al. } \\
\text { (2001) }\end{array}$ & $\begin{array}{c}\text { Membrana de } \\
\text { Polietersulfona (PES) e } \\
\text { Óxido de Alumínio } \\
\left(\mathrm{Al}_{2} \mathrm{O}_{3}\right) .\end{array}$ & $\begin{array}{l}\text { Utilização do método de } \\
\text { inversão de fase para } \\
\text { preparação de membranas de } \\
\text { fibra ocas inorgânicas de } \\
\text { óxido de alumínio }\left(\mathrm{Al}_{2} \mathrm{O}_{3}\right) \text {. }\end{array}$ & $\begin{array}{l}\text { - As membranas com estrutura } \\
\text { assimétrica, com forma de } \\
\text { sponge no centro, e fingers nas } \\
\text { paredes externa e interna da } \\
\text { fibra; } \\
\text { - Melhora da resistência } \\
\text { mecânica pela adição das } \\
\text { partículas menores; } \\
\text { - À medida que a razão } \\
\mathrm{Al}_{2} \mathrm{O}_{3} / \text { PESf aumenta, tanto o } \\
\text { tamanho dos poros quanto a } \\
\text { porosidade diminuem. }\end{array}$ \\
\hline $\begin{array}{l}\text { Liang et } \\
\text { al. (2012) }\end{array}$ & $\begin{array}{c}\text { Polietersulfona } \\
\text { (PES)/Montimorilonita } \\
\text { (MMT) e } \\
\text { Polietersulfona/Dióxido } \\
\text { de Titânio }\left(\mathrm{TiO}_{2}\right) \text {. }\end{array}$ & $\begin{array}{l}\text { Preparação de membranas } \\
\text { PES/MMT e PES/TiO2 para } \\
\text { permeações de } \mathrm{CO}_{2} \text { e } \mathrm{CH}_{4} \text {. }\end{array}$ & $\begin{array}{c}\text { - Para PES/MMT, as } \\
\text { permeabilidades de } \mathrm{CO}_{2} \text { e } \mathrm{CH}_{4} \\
\text { aumentaram com o conteúdo } \\
\text { de MMT; } \\
\text { - } \quad \text { Para } \mathrm{PES} / \mathrm{TiO} 2 \text { a seletividade } \\
\text { de } \mathrm{CO}_{2} / \mathrm{CH}_{4} \text { aumentou com } \\
\text { menores teores de } \mathrm{TiO}_{2} \mathrm{e} \\
\text { depois diminuiu com um } \\
\text { aumento adicional na de } \mathrm{TiO} 2 .\end{array}$ \\
\hline $\begin{array}{l}\text { Ferreira et } \\
\text { al. (2019) }\end{array}$ & $\begin{array}{l}\text { Membranas de } \\
\text { Polietersulfona/Argila } \\
\text { Natural montmorilonita. }\end{array}$ & $\begin{array}{l}\text { Produzir membranas de } \\
\text { polietersulfona/argila natural a } \\
\text { fim de modificar a morfologia, } \\
\text { obtendo membranas eficientes } \\
\text { para aplicação no tratamento } \\
\text { de efluentes da indústria têxtil. }\end{array}$ & $\begin{array}{l}\text { A adição de argila resultou } \\
\text { uma estrutura com poros, } \\
\text { macroporos e fingers em todo } \\
\text { o suporte poroso; } \\
\text { A presença de argila } \\
\text { proporcionou maior } \\
\text { rendimento, pois a separação } \\
\text { indigo blue (poluente da } \\
\text { indústria têxtil) apresentou } \\
94,0 \% \text { para as membranas } \\
\text { contendo } 1 \% \text { de argila. }\end{array}$ \\
\hline $\begin{array}{l}\text { Medeiros } \\
\text { et al. } \\
(2020)\end{array}$ & $\begin{array}{l}\text { Membranas de } \\
\text { Polietersulfona } \\
\text { Polietersulfona/ } \\
\text { Montmorilonita. }\end{array}$ & $\begin{array}{l}\text { Investigar a influência de } \\
\text { parâmetros na estrutura e } \\
\text { propriedades de } \\
\text { polietersulfona e } \\
\text { polietersulfona/argila, com o } \\
\text { auxílio do planejamento } \\
\text { experimental. }\end{array}$ & $\begin{array}{l}\text { - Por meio do planejamento } \\
\text { experimental, concluiu-se que } \\
\text { a membrana com } 16 \% \text { de } \\
\text { argila apresentou a melhor } \\
\text { composição, considerando suas } \\
\text { características e propriedades. }\end{array}$ \\
\hline Floros et & $\begin{array}{c}\text { Membranas de } \\
\text { Politetrafluoroetileno } \\
\text { (PTFE)/Poli(álcool }\end{array}$ & $\begin{array}{c}\text { Avaliar o desempenho no } \\
\text { processo de dessalinização } \\
\text { (MD), examinando a eficácia } \\
\text { da deposição de PVA } \\
\text { qualitativa e quantitativamente } \\
\text { por meio de ângulo de } \\
\text { contato, cálculos de tamanho }\end{array}$ & $\begin{array}{l}\text { - Os resultados da caracterização } \\
\text { mostraram que devido ao } \\
\text { revestimento de PVA, as } \\
\text { membranas modificadas } \\
\text { apresentaram hidrofobia } \\
\text { reduzida, menor LEP, BubP e } \\
\text { porosidade; } \\
\text { - A avaliação da dessalinização }\end{array}$ \\
\hline
\end{tabular}




\begin{tabular}{|c|c|c|c|}
\hline al. (2020) & vinílico) (PVA). & $\begin{array}{c}\text { de poro, porosidade, pressão } \\
\text { de entrada de líquido (LEP) e } \\
\text { pressão de ponto de bolha } \\
\text { (BubP). }\end{array}$ & $\begin{array}{l}\text { das membranas modificadas } \\
\text { mostrou um aumento notável } \\
\text { ( } 2,7 \text { vezes) no fluxo de água; } \\
\text { mantendo uma alta eficiência. }\end{array}$ \\
\hline
\end{tabular}

Fonte: Autores.

\section{Conclusão}

As membranas do tipo fibra oca apresentam uma ótima alternativa para os processos de separações clássicos, tendo uma grande versatilidade de aplicações em diferentes áreas, como a médica, química, alimentícia, tratamento de águas, tratamento de despejos industriais e biotecnologia. Vários polímeros e materiais inorgânicos podem ser utilizados para a fabricação de membranas de fibra oca através do método de inversão de fases, como a polietersulfona, polisulfona, acetato de celulose dentre os polímeros, e carbeto de silício, alumina e níquel dentre os materiais inorgânicos. Diante das várias vantagens oferecidas pela utilização de fibra oca nos processos de separação, a relação entre a área de permeação e o volume do módulo ser muito maior quando comparada com as outras geometrias, além do fato de apresentarem uma densidade de empacotamento alta devido ao diâmetro das fibras, tornando auto suportadas, faz com que certas configurações de separação sejam alcançadas, e a fibra oca seja ideal para certas aplicações.

Como sugestão para trabalhos futuros, poderia ser apresentado uma pesquisa onde fosse possível verificar os estudos de membranas com diversas camadas de materiais. Sendo assim, seria possível levantar dados sobre os avanços no que se diz respeito a membranas híbridas, com camadas seletivas de diferentes materiais. Também se faz uma sugestão a respeito de uma revisão da literatura sobre a comparação da eficiência real de vários tipos de membranas (orgânicas e inorgânicas) em determinados efluentes.

\section{Agradecimentos}

Os autores agradecem ao Laboratório de Desenvolvimento e Caracterização de Membranas/CCT/UFCG - LDCM, ao Conselho Nacional de Desenvolvimento Científico e Tecnológico - CNPq e à Coordenação de Aperfeiçoamento de Pessoal de Nível Superior - CAPES, pelo apoio financeiro.

\section{Referências}

Anadão, P. (2010). Ciência e Tecnologia de Membranas. Artliber Editora.

Armoa, M. H. \& Jafelicci Jr, M. (2011) Princípios e aplicações de processos de separação por membranas inorgânicas. Ciência \& Tecnologia, 2 (1), 80-97.

Baker, R. W. (2004). Membrane Technology and Applications. (2a ed.). John Wiley \& Sons Ltd.

Bertoldo, L. C. Desenvolvimento de Membranas de Fibras Ocas para Ultrafiltração e Nanofiltração Utilizando Imersão em Banho Duplo de Precipitação. Dissertação de Mestrado (Engenharia Química) - Programa de Pós-Graduação em Engenharia, COPPE, Universidade Federal do Rio de Janeiro, Rio de Janeiro, 2005.

De Wit, P.; Van Daalen, F. S. \& Benes, N. E. (2017). The mechanical strength of a ceramic porous hollow fiber. Journal of Membrane Science, 524, 721-728.

Drioli, E. \& Giorno, L. (2010). Comprehensive membrane science and engineering. Newnes.

Ferreira, R. S. B., Salviano, A. F., Oliveira, S. S. L., Araújo, E. M., Medeiros, V. N. \& Lira, H. L. (2019). Treatment of Effluents from the Textile Industry through Polyethersulfone Membranes. Water, 11(12), 2540, 2019.

Floros, I. N., Kouvelos, E. P., Pilatos, G. I., Hadjigeorgiou, E. P., Gotzias, A. D., Favvas, E. P., \& Sapalidis, A. A. (2020). Enhancement of Flux Performance in PTFE Membranes for Direct Contact Membrane Distillation. Polymers, 12(2), 345.

Galvão, D. F. \& Gomes, E. R. S. (2015). Os processos de separação por membranas e sua utilização no tratamento de efluentes industriais da indústria de laticínios: revisão bibliográfica. Revista do Instituto de Laticínios Cândido Tostes, 70(6), 349-360. 
Guillen, G. R., Pan, Y., Li, M. \& Hoek, E. M. V. (2011) Preparation and characterization of membranes formed by nonsolvent induced phase separation: A review. Industrial \& Engineering Chemistry Research, 50, $3798-3817$.

Habert, A. C., Borges, C. P. \& Nóbrega, R. (2006). Processos de separação por membranas. E-papers.

Hamza, A., Pham, V. A., Matsuura, T. \& Santerre, J. P. (1997). Development of membranes with low surface energy to reduce the fouling in ultrafiltration applications. Journal of Membrane Science, 131, 217-227.

Hilal, N., Ismail, A. F. \& Wright, C. J. (2015). Membrane Fabrication. CRC Press, Taylor and Fracis Group.

Khulbe, K. C. \& Matsuura, T. (2016). Recent progress in polymeric hollow-fibre membrane preparation and applications. Membrane Technology, 2016(7), 7 13 .

Khulbe, K. C., Feng, C. Y. \& Matsuura, T. (2008). Synthetic Polymeric Membranes: Characterization by Atomic Force Microscopy. Springer Science \& Business Media.

Kingsbury, B. F. K. \& Li, K. (2009). A morphological study of ceramic hollow fibre membranes. Journal of Membrane Science, 328(1), 134-140.

Kojuch, L. R. Obtenção de Membranas para Separação de água-óleo a partir de Nanocompósitos de Poliamida 6.6. Monografia (ANP/PRH-25) Departamento de Engenharia de Materiais da Universidade Federal de Campina Grande, Campina Grande, 2010.

Huang, A. \& Feng, B. (2018) Synthesis of novel graphene oxide-polyimide hollow fiber membranes for seawater desalination. Journal of Membrane Science, $548,59-65$.

Lautenschlager, R. S., Ferreira Filho, S. S. \& Pereira, O. (2009). Modelação matemática e otimização operacional de processos de membrana de ultrafiltração. Revista de Engenharia Sanitária e Ambiental, 14(2), 215-222.

Lavezo, A. E. Estudo da minimização do custo de um processo de separação de misturas gasosas multicomponentes atraves da membrana de fibra oca. Dissertação (Mestrado em Engenharia Química). Universidade Estadual de Campinas, 2011.

Li, N. N., Fane, A. G., Ho, W. S. W. \& Matsuura, T. (2008) Advanced Membrane Technology and Applications. $1^{\text {a }}$ edição. New Jersey: John Wiley \& Sons.

Liang, C. Y., Uchytil, P., Petrychkovych, R., Lai, Y. C., Friess, K., Sipek, M., Reddy, M. M. \& Suen, S. Y. (2012). A comparison on gas separation between PES (polyethersulfone)/MMT (Na-montmorillonite) and PES/TiO2 mixed matrix membranes. Separation and Purification Technology, 92, 57-63.

Lima, R. C. O, Lira, H. L, Neves, G. A, Silva, M. C \& Silva, C. D. (2011). Aproveitamento do Resíduo de Serragem de Granito para Fabricação de Membranas Cerâmicas de Baixo Custo. Revista Eletrônica de Materiais e Processos, 6(3), 163-169.

Liu, S. \& Gavalas, G. R. (2005). Oxygen selective ceramic hollow fiber membranes. Journal of Membrane Science, 246(1), 103-108.

Mack, J. J., Cox, B. N., Sudre, O., Corrin, A. A., Lucato, S. L. D. S., Ma, C. \& Andrew, J. S. (2009). Achieving nutrient pumping and strain stimulus by magnetic actuation of tubular scaffolds. Smart Materials and Structures, 18(10), 104025.

Madsen, B. R. Characterization and physicochemical modifications of polymer hollow fiber membranes for biomedical and bioprocessing applications. Dissertação (Engenharia Biológica e Irrigacional) - Utah State University, Logan 2010.

Mahon, H. I. Permeability separatory apparatus and process utilizing hollow fibers. U.S. Patent n. 3,228,877, 1966.

Maldonado, J. (1991). Membranas e processos de Separação. Rio de Janeiro: DEPM/CTM/INT.

Medeiros, V. D. N., Silva, B. I. A., Ferreira, R. D. S. B., Oliveira, S. S. L., Dias, R. A. \& Araújo, E. M. (2020) Optimization of Process Parameters for Obtaining Polyethersulfone/Additives Membranes. Water, 12(8), 2180.

Mulder, M. (1996). Basic Principles of Membrane Technology. (2a ed.). Kluwer Acad. Pub.

Nath, K. (2017). Membrane separation processes. (2a ed.). Delhi: PHI Learning Pvt. Ltd.

Pereira, A.S., Shitsuka D. M., Parreira, F. J., Shitsuka, R. (2018). Metodologia da pesquisa científica. UFSM.

Peng, N., Widjojo, N., Sukitpaneenit, P., Teoh, M. M., Lipscomb, G. G., Chung, T. S. \& Lai, J. Y. (2012). Evolution of polymeric hollow fibers as sustainable technologies: Past, present, and future. Progress in Polymer Science, 37, 1401-1424.

Porter, M C. (1989). Handbook of industrial membrane technologyNoyes Publications.

Radjenović, J., Matošić, M., Mijatović, I., Petrović, M. \& Barceló, D. (2007) Membrane bioreactor (MBR) as an advanced wastewater treatment technology. Handbook Environmental Chemistry, 5, 37-101.

Rastegarpanah, A. \& Mortaheb, H. R. (2016). Surface treatment of polyethersulfone membranes for applying in desalination by direct contact membrane distillation. Desalination, 377, 99-107.

Rodrigues, J. S., Cordeiro, J., Calazans, G. M., Cordeiro, J. L., \& Guimarães, J. C. S. (2018). Presença de fármacos e hormônios na água: uma análise cienciométrica. Research, Society and Development, 7(6), e776185-e776185.

Schneider, R. P. \& Tsutiya, M. T. (2001). Membranas filtrantes para o tratamento de água, esgoto e água de reusoAssociação Brasileira De Engenharia Sanitária. 
Research, Society and Development, v. 10, n. 1, e55910111206, 2021

(CC BY 4.0) | ISSN 2525-3409 | DOI: http://dx.doi.org/10.33448/rsd-v10i1.11206

Shamu, A., Dunnewold, M., Miedema, H., Borneman, Z. \& Nijmeijer, K. (2019). Permeation of supercritical CO2 through dense polymeric membranes. The Journal of Supercritical Fluids, 144, 63-70.

Silva, M. C., Lira, H. L., Lima, R. C. O. \& Freitas, N. L. (2015). Effect of Sintering Temperature on Membranes Manufactured with Clays for Textile Effluent Treatment. Advances in Materials Science and Engineering, 1-7.

Tan, X., Liu, S. \& Li, K. (2001). Preparation and characterization of inorganic hollow fiber membranes. Journal of Membrane Science, 188(1), 87-95.

Wang, Y., Zhu, J., Dong, G., Zhang, Y., Guo, N. \& Liu, J. (2015). Sulfonated halloysite nanotubes/polyethersulfone nanocomposite membrane for efficient dye purification. Separation and Purification Technology, 150, 243-251.

Way, J. D. \& Roberts, D. L. (1992). Hollow fiber inorganic membranes for gas separations. Separation science and technology, 27(1), 29-41. 